\title{
Adherencia terapeútica y factores condicionantes en su cumplimiento en pacientes con tuberculosis pulmonar atendidos en la Microred la Palma, Ica 2017
}

\author{
Therapeutic adherence and conditioning factors in its compliance in patients \\ with pulmonary tuberculosis attended at the Microred Ia Palma, Ica 2017
}

\footnotetext{
Meza-Condezo Walter ${ }^{1, a}$, Peralta-Pumapillo Adderly,a, Quispe-Gómez Freddy, ${ }^{1, a}$, Cáceres-Bellido Fermín ${ }^{1,2, a}$

1. Universidad Nacional San Luis Gonzaga de Ica

2. Hospital Santa María del Socorro de Ica, Perú.

a. Médico Cirujano

b. Cirujano General
}

\section{Correspondencia:}

Meza Condezo Walter

Domicilio: Calle Los Pacaes A16

Teléfono: 920646879

Email:

waltermezacondezo@gmail.com

Contribuciones De Autoría:

WMC, APP, FQG, y FCB:

Contribuciones en la concepción y diseño del manuscrito, recolección, análisis e interpretación de los datos; redacción y revisión crítica del contenido del manuscrito y la aprobación final del artículo.

Conflicto De Intereses: No declarados.

Financiamiento: Autofinanciado.

\section{Como Citar}

Meza-Condezo W, Peralta-Pumapillo A, Quispe-Gómez F, Cáceres-Bellido Fermín E. Adherencia terapéutica y factores condicionantes en su cumplimiento en pacientes con tuberculosis pulmonar atendidos en la microred la palma, Ica 2017. Rev méd panacea. 2018; 7(1): 22-27

Recibido: 03 - 03 - 2018

Aceptado: 20 - 03 - 2018

Publicado: 23 - 04 - 2018

\section{RESUMEN}

Objetivo: Determinar la adherencia terapéutica y factores condicionantes en su cumplimiento en pacientes con tuberculosis pulmonar atendidos en la Micro Red La Palma, Ica 2017. Materiales y métodos: Nivel de investigación observacional, tipo analítico, prospectivo y transversal. Realizado mediante un test de Morisky-Green-Levine. El análisis se realizó mediante spss 24.0 aplicando el test exacto de fisher ( $p$-valor $<0.05$ ) Resultados: Se encuentra que existe $42 \%$ de mala adherencia, entre factores asociados, se encuentra con los factores relacionados a la terapéutica, con relación a la asistencia sanitaria, y con factores en relación al paciente.

Palabras clave: Tuberculosis, tratamiento farmacológico, cumplimiento de la medicación. (Fuente: DeCS BIREME).

\section{ABSTRACT}

Objective: To determine the therapeutic adherence and conditioning factors in its compliance in patients with pulmonary tuberculosis treated in the La Palma Micro-Network, Ica 2017. Materials and methods: Level of observational research, analytical, prospective and transversal type. Performed by a Morisky-Green-Levine Test. The analysis was performed by SPSS 24.0 applying the Fisher exact test ( $p$-value $<0.05$ ) Results: It is found that there is $42 \%$ of poor adherence, among associated factors, it is found with the factors related to the therapeutic, in relation to health care, and with factors in relation to the patient.

Keywords: Tuberculosis, drug therapy, medication Adherence. (Source: MeSH NLM) 


\section{INTRODUCCIÓN}

En el Perú cada año existen aproximadamente 29 mil casos nuevos de personas con enfermedad activa y 17 mil casos nuevos de tuberculosis pulmonar encontrándose la presencia de bacterias de la tuberculosis en la baciloscopía considerándose como frotis positivo, es importante debido que es tarea fundamental que el médico realice las indicaciones respectivas adecuadas ya que de ello dependerá el comportamiento del paciente frente a la adherencia farmacológica, las indicaciones recibidas implica saber: cuando y que debe hacer, a su vez es importante lograr que los pacientes logren adquirir y mantener la adherencia al tratamiento cumpliendo con todo lo que se le indica referente a su salud.

La tuberculosis es considerada por la Organización Mundial de la Salud como una de las enfermedades que afecta a la tercera parte de la población mundial, siendo una enfermedad frecuente en países en desarrollo y en especial en las áreas más densamente pobladas (1).

A nivel mundial la tuberculosis es la segunda causa de mortalidad, después del SIDA, en el 2013, se estima que se contrajeron tuberculosis multidrogorresistente 450 000 personas, y que 170000 fallecieron por esta causa. (1)

La tuberculosis en el Perú ocupa el décimo quinto lugar de las causas de muerte (2), y el vigésimo séptimo puesto de carga de enfermedad medida por años de vida saludable perdidos (AVISA) (3). Afecta, predominantemente, a los estratos sociales más pobres de las grandes ciudades del país (4). Las tasas notificadas de incidencia (casos nunca tratados por cada 100 mil habitantes) y de morbilidad total (nuevos y antes tratados por cada 100 mil habitantes) han disminuido entre 2 a 3\% por año entre los años 2011 a 2015, de 97,4

\section{a 87,6 en incidencia y de 109,7 a 99,5 en morbilidad.}

Según el Ministerio de Salud del Perú, la tuberculosis es un grave problema de salud pública en el Perú, esto debido por muchos factores, entre los más importantes, debido a la pobre adherencia al tratamiento que presentan las personas que padecen dicha enfermedad (5). En esta investigación pretendemos determinar la adherencia terapéutica y factores condicionantes en su cumplimiento en los pacientes con tuberculosis pulmonar que son atendidos en la Microred La Palma, Ica 2017.

\section{MATERIALES Y MÉTODOS}

El tipo de estudio de la investigación fue Observacional, de tipo prospectivo obteniéndose a partir entrevistas con los pacientes que tuviesen tuberculosis, de corte Transversal; descritos en un solo momento y espacio en cada uno de los estudiantes; estudio de tipo Analítico, donde el propósito es evidenciar una variable independiente (adherencia terapéutica) en relación a una variable dependiente (los factores condicionantes en su cumplimiento, tuberculosis pulmonar), más no interviene en la historia natural de la enfermedad. La población de estudio estuvo conformada por 52 pacientes de la Micro red La Palma: 15 pacientes en el C. S. La Palma, 20 pacientes del P.S. Sr de Lúren, 11 del C. S. San Joaquín, 2 pacientes del P.S. Cachiche y 4 pacientes del PS.S. Huarango, los cuales son tomados a través del muestreo no probabilístico y por conveniencia.

\section{RESULTADOS}

Entre los pacientes encuestados con tuberculosis pulmonar, se le encontró edades comprendidas entre, 01 año y 74 años, obteniendo una mediana de 28 años, siendo la edad más frecuente de 22 años.

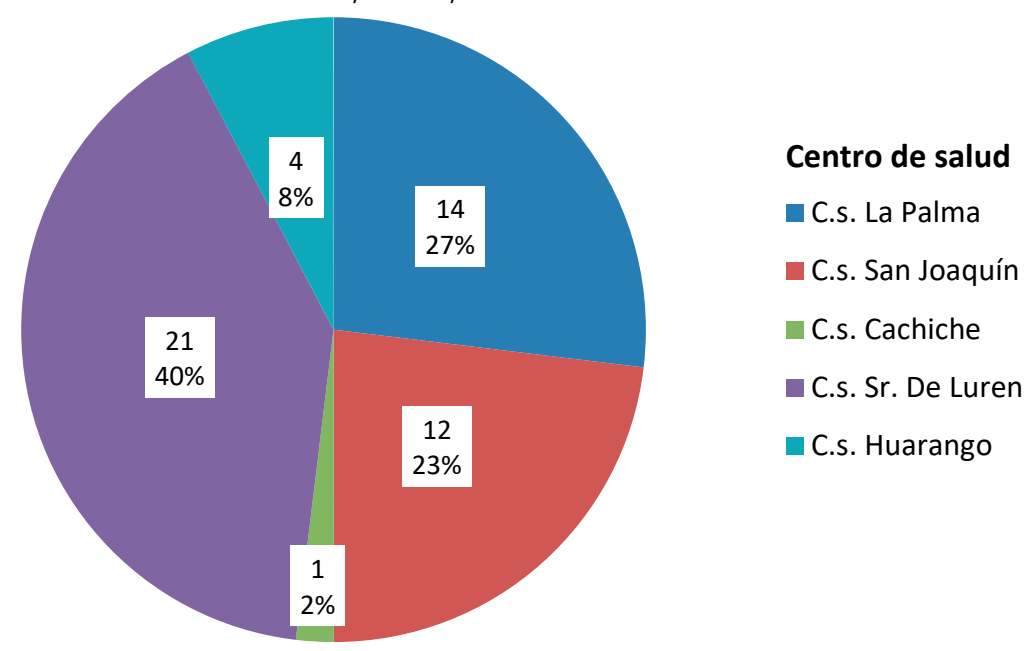

Gráfico 1. Distribución de pacientes por establecimiento de Salud.

Se entrevistaron a 52 pacientes con el diagnóstico de tuberculosis pulmonar, que pertenecen a la Micro Red La Palma, donde el $40.38 \%$ pertenece al PS. Luren, 26.92\% al C.S. La Palma 23.08\% al C.S. San Joaquín, 7.69\% al PS. Huarango y con menor proporción al PS. Cachiche. 
Tabla 1. Resultados del test de Morisky-Green-Levine, para determinar el cumplimiento a la medicación

\begin{tabular}{|c|c|c|c|c|c|c|c|}
\hline & \multirow{2}{*}{ Resp. Adh } & \multicolumn{2}{|c|}{ Sí } & \multicolumn{2}{|c|}{ No } & \multicolumn{2}{|c|}{ Total } \\
\hline & & $\mathbf{N}$ & $\%$ & $\mathbf{N}$ & $\%$ & $\mathbf{N}$ & $\%$ \\
\hline $\begin{array}{l}\text { 1. ¿Se olvida alguna vez de tomar sus } \\
\text { medicamentos? }\end{array}$ & No & 11 & $21,2 \%$ & 41 & $78,8 \%$ & 52 & $100,0 \%$ \\
\hline $\begin{array}{l}\text { 2. ¿Toma sus medicamentos a la hora } \\
\text { indicada? }\end{array}$ & SI & 32 & $61,5 \%$ & 20 & $38,5 \%$ & 52 & $100,0 \%$ \\
\hline $\begin{array}{l}\text { 3. Cuando se encuentra bien, ¿deja } \\
\text { alguna vez de tomarlos? }\end{array}$ & NO & 10 & $19,2 \%$ & 42 & $80,8 \%$ & 52 & $100,0 \%$ \\
\hline $\begin{array}{l}\text { 4. Si alguna vez le caen mal, ¿deja de } \\
\text { tomar la medicación? }\end{array}$ & NO & 11 & $21,2 \%$ & 41 & $78,8 \%$ & 52 & $100,0 \%$ \\
\hline
\end{tabular}

De los 52 encuestados, para el tratamiento médico y farmacológico contra la tuberculosis, donde el $21.2 \%$ (11 pacientes) olvidan tomar sus medicamentos, el 38.5\% (20 pacientes) no toma sus medicamentos a la hora indicada, el $19.2 \%$ ( 10 pacientes) deja de tomar los medicamentos cuando se encuentra bien y el $21.2 \%$ (11 pacientes) deja de tomar los medicamentos cuando no se encuentra bien, como se muestra en la Tabla 1.

Tabla 2. Adherencia terapéutica según el test de Morisky-Green-Levine

\begin{tabular}{lcc} 
& N & $\%$ \\
No adherente & 22 & 42,3 \\
Adherente al tratamiento & 30 & 57,7 \\
Total & 52 & 100,0 \\
\hline
\end{tabular}

Siendo según el test evaluado, para el incumplimiento al tratamiento farmacológico de los pacientes, donde el $42.3 \%$ (22 pacientes) no tiene adherencia terapéutica al esquema de tuberculosis, como se observa en la tabla 2.

Tabla 3. Test $X 2$ de independencia, asociación entre los factores relacionado a la terapéutica con la adherencia terapéutica.

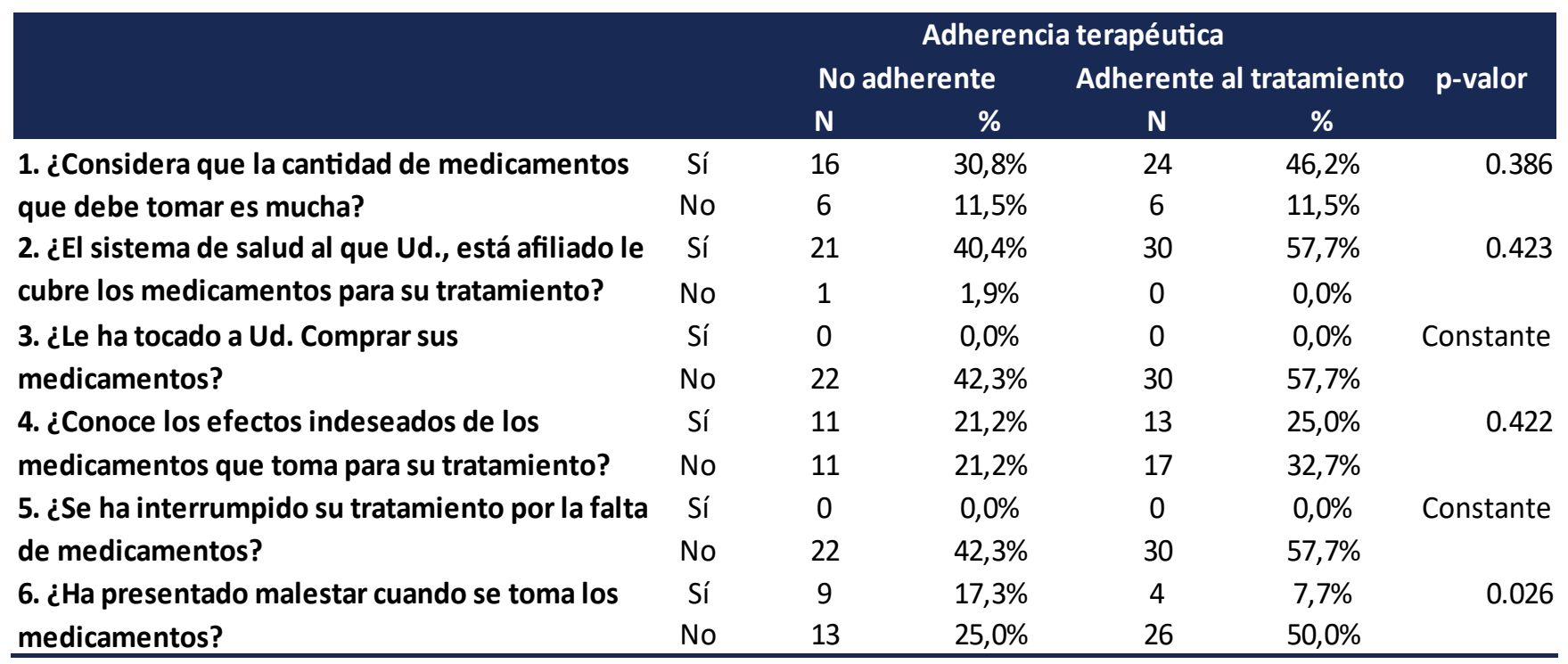

Con respecto al factor condicionante relacionados a la terapéutica en relación de la adherencia y terapéutica, no muestran asociación ( $p$-valor >0.05), ni con la percepción de tomar muchos medicamentos, ni sobre el impedimento de tener los medicamentos por sus seguro, ni con la compra de medicamentos, ni con la interrupción de la terapia por falta de medicamentos, pero si muestra asociación con la percepción de molestias al tomar los medicamentos, Tabla 3. 
Tabla 4. Test X2 de independencia, asociación entre los factores relacionados con la enfermedad con la adherencia terapéutica.

\begin{tabular}{llccccc} 
& \multicolumn{7}{c}{ Adherencia terapéutica } \\
& & No adherente & Adherente al tratamiento & p-valor \\
& & N & $\%$ & N & $\%$ & \\
\hline 7. ¿La tos es frecuente? & Sí & 4 & $7,7 \%$ & 4 & $7,7 \%$ & 0.459 \\
& No & 18 & $34,6 \%$ & 26 & $50,0 \%$ & \\
8. ¿'Ha tenido fracaso en & Sí & 2 & $3,8 \%$ & 0 & $0,0 \%$ & 0.174 \\
tratamientos anteriores? & No & 20 & $38,5 \%$ & 30 & $57,7 \%$ & \\
\hline
\end{tabular}

Con los factores condicionantes relacionados con la enfermedad, no se encontró asociación con la adherencia terapéutica; ni con la sintomatología ni con fracasos de esquemas anteriores ( $p$-valor >0.05), Tabla 4.

Tabla 5. Test $\mathrm{X} 2$ de independencia, asociación entre los factores relacionados con el sistema de asistencia sanitaria con la adherencia terapéutica.

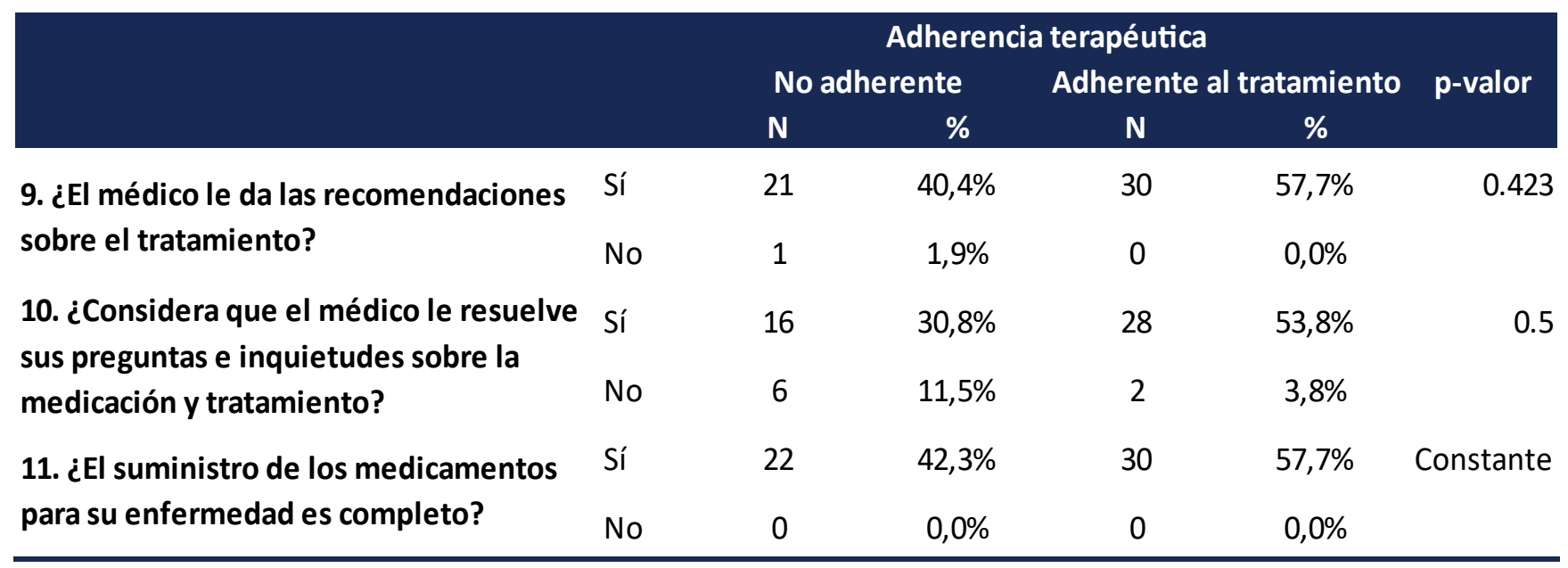

Sobre los factores relacionados con el sistema de asistencias sanitaria y adherencia terapéutica, no presenta asociación ( $p$-valor $>0.05$ ) con las recomendaciones que brinda el médico, ni con el suministro completo de los medicamentos para su terapia, más si tiene asociación sobre las inquietudes que resuelve el médico sobre la medicación $(p \leq 0.05)$, Tabla 5 .

Tabla 5. Test $X 2$ de independencia, asociación entre los factores relacionados con el sistema de asistencia sanitaria con la adherencia terapéutica.

\begin{tabular}{|c|c|c|c|c|c|c|}
\hline & \multicolumn{6}{|c|}{ Adherencia terapéutica } \\
\hline & & \multicolumn{2}{|c|}{ No adherente } & \multicolumn{2}{|c|}{ Adherente al tratamiento } & \multirow[t]{2}{*}{ p-valor } \\
\hline & & $\mathbf{N}$ & $\%$ & $\mathbf{N}$ & $\%$ & \\
\hline $\begin{array}{l}\text { 12. ¿Cree que no necesita } \\
\text { seguir el tratamiento para el }\end{array}$ & Sí & 0 & $0,0 \%$ & 3 & $5,8 \%$ & 0.184 \\
\hline manejo de su enfermedad? & No & 22 & $42,3 \%$ & 27 & $51,9 \%$ & \\
\hline $\begin{array}{l}\text { 13. ¿Se ha sentido } \\
\text { motivado/da a cumplir con }\end{array}$ & Sí & 16 & $30,8 \%$ & 28 & $53,8 \%$ & 0.5 \\
\hline el tratamiento? & No & 6 & $11,5 \%$ & 2 & $3,8 \%$ & \\
\hline $\begin{array}{l}\text { 14. ¿Cree Ud. Que la } \\
\text { enfermedad lo ha limitado }\end{array}$ & Sí & 20 & $38,5 \%$ & 22 & $42,3 \%$ & 0.107 \\
\hline en sus actividades diarias? & No & 2 & $3,8 \%$ & 8 & $15,4 \%$ & \\
\hline
\end{tabular}


Tabla 4: Descripción de los conocimientos correctos e incorrectos frente al VIH-SIDA en los estudiantes de una universidad nacional de Ica, 2017.

\begin{tabular}{|c|c|c|c|c|c|c|}
\hline $\begin{array}{l}\text { 15. Debido a la enfermedad, } \\
\text { ¿ha notado cambio en su }\end{array}$ & Sí & 22 & $42,3 \%$ & 27 & $51,9 \%$ & 0.184 \\
\hline estilo de vida? & No & 0 & $0,0 \%$ & 3 & $5,8 \%$ & \\
\hline $\begin{array}{l}\text { 16. ¿Conoce la dieta a seguir } \\
\text { para cumplir con el }\end{array}$ & Sí & 12 & $23,1 \%$ & 18 & $34,6 \%$ & 0.456 \\
\hline tratamiento? & No & 10 & $19,2 \%$ & 12 & $23,1 \%$ & \\
\hline \multirow{2}{*}{$\begin{array}{l}\text { 17. ¿Recibe apoyo de su } \\
\text { familia? }\end{array}$} & Sí & 14 & $26,9 \%$ & 26 & $50,0 \%$ & 0.054 \\
\hline & No & 8 & $15,4 \%$ & 4 & $7,7 \%$ & \\
\hline $\begin{array}{l}\text { 18. ¿Pertenece a algún } \\
\text { programa de seguimiento o }\end{array}$ & Sí & 19 & $36,5 \%$ & 27 & $51,9 \%$ & 0.506 \\
\hline apoyo en el tratamiento? & No & 3 & $5,8 \%$ & 3 & $5,8 \%$ & \\
\hline \multirow{2}{*}{$\begin{array}{l}\text { 19. ¿Queda lejos de su casa } \\
\text { el Centro de salud? }\end{array}$} & Sí & 8 & $15,4 \%$ & 10 & $19,2 \%$ & 0.525 \\
\hline & No & 14 & $26,9 \%$ & 20 & $38,5 \%$ & \\
\hline
\end{tabular}

Y sobre los factores relacionados con el paciente y la adherencia terapéutica, en la investigación, no muestra asociación ( $p$-valor >0.05), con variables como: la negación ante el seguimiento del tratamiento, sobre la limitación que ejerce la enfermedad sobre las actividades diarias, o cambio de estilos de vida, conocimiento sobre dieta durante el tratamiento, apoyo familiar, pertenecer en algún grupo de apoyo, o sobre la lejanía del establecimiento de salud para recibir o continua tratamiento. Pero se encuentra relación a la adherencia terapéutica con la motivación a cumplir con el tratamiento, Tabla 6.

\section{DISCUSIÓN}

La tuberculosis es considerada por la Organización Mundial de la Salud como una de las enfermedades que afecta a la tercera parte de la población mundial, y es un gran problema de salud pública (1). Donde uno de sus problemas arraiga en el mal tratamiento que siguen los pacientes, y esto por diversos motivos, siendo este el motivo de la investigación.

Se identifican casos entre pacientes con 01 año hasta 74 años de edad, con una media de 18 años, siendo la edad más frecuente de 22 años, datos que son compartidos al igual que Iparraguirre (6), donde su estudio realizado en Trujillo, el $74.47 \%$ de los pacientes tenían edades entre 20 a 65 años, con una edad promedio entre 10-19 años, con mayor frecuencia en el sexo masculino, González (7), en su estudio realizado en Ecuador, encontró que existe mayor incidencia en grupo de 39 - 50 años de edad, siendo su prevalencia mayor de 40 años, donde se explica que a partir de esta edad, el organismo empieza a disminuir sus defensas, haciéndose más sensible a adquirir este tipo de enfermedades infecciosas.

Al identificar la adherencia terapéutica, mediante el Test de Morisky-Green-Levine (8) se encuentra que el $21.2 \%$ olvida tomar sus medicamentos, $38.5 \%$ no toma sus medicamentos a la hora indicada, $19.2 \%$ deja de tomar los medicamentos cuando se encuentra bien y el $21.2 \%$ deja de tomar los medicamentos cuando no se encuentra bien, esto deja como resumen que el $42.3 \%$ no tiene adherencia terapéutica al esquema de tuberculosis, estos resultados frente a diversos estudios nacionales, mantienen diversos porcentajes, tal es el estudio de Iparraguirre (6), donde presenta un $87.23 \%$ de adherencia farmacológica media, y solo un $2.13 \%$ de adherencia baja, Suárez y Catherine (5), muestran en Lima, que $46 \%$ de la población presenta una adherencia con nivel medio, Murcia (9), al evaluar a sus pacientes en el inicio y al final del tratamiento, muestra que se cumplió con un $83.5 \%$, siendo $75 \%$ mayor en mujeres, a nivel internacional, González (7), muestra que en sus pacientes una adherencia del $89 \%$, de un tratamiento entre 6 a 12 meses, siendo diversos motivos del abandono. El estudio de Garnica y cols. (10), determina que 7 de cada 13 pacientes esta adherido al tratamiento del programa Nacional de Control de Tuberculosis, lo cual representa aun cifras alarmantes.

En la evaluación entre la adherencia terapéutica del esquema de tuberculosis y variables de aspecto generales, no se encontró asociación ( $p$-valor >0.05) entre la edad, el estado civil, antecedente de tuberculosis familiar, personas que habitan en el mismo dormitorio, grado de instrucción, afiliación al seguro integral de salud, hábito de drogadicción o tabaco, o presencia de personas con VIH. Resultados presentados que se muestran en la localidad, pero que en otros estudios encuentran relación con el sexo, grado de instrucción y lugar de procedencia, mas no con la edad, o estado civil o ingreso económico, donde los autores Araujo, Arévalo, y Torres (11), muestran que los factores biosociales son importante en la adherencia al tratamiento, Murcia (8), o Zeledón y cols. (12), encuentran que tener una primaria incompleta o ser de sexo masculino respectivamente, presentan un factor de riesgo, lo cual debe tener importancia y ser de vigilancia con la finalidad de 
contribuir a minimizar la aparición de tuberculosis multidrogoresistente.

A la relación entre la adherencia terapéutica y factores relacionados a la terapéutica, se encuentra asociación con la percepción de molestias al tomar los medicamentos, con los factores relacionados con la enfermedad tampoco muestra alguna asociación. Con los factores relacionados al sistema de asistencia sanitaria, se encuentra relación, con la resolución de inquietudes por parte del médico acerca de la medicación, a esto Zeledón y cols. $(12,9)$, manifiesta que el abandono del paciente, se debe al desconocimiento del esquema de tratamiento y mala relación con el personal de salud, el cual es un punto prioritario en la aceptación del paciente, los autores Navarro, Rueda y Mendoza (13), en su estudio realizado en Venezuela, coinciden que un factor asociado en la mala adherencia terapéutica es el desconocimiento de la importancia de

\section{REFERENCIAS BIBLIOGRÁFICAS}

1. Organización Mundial de la Salud. WHO | World TB Day, 24 March 2018 [Internet]. WHO. 2018 [citado 23 de marzo de 2018]. Disponible en: http://www.who.int/campaigns/tb-day/2018/en/

2. Ministerio de Salud, Oficina General de Tecnologías de la Información. Principales causas de mortalidad por sexo Perú, año 2014 [Internet]. 2015. Disponible en:

http://www.minsa.gob.pe/estadisticas/estadisticas/ mortalidad/macros.asp?00

3. Ministerio de Salud-Dirección general de Epidemiología. Carga de enfermedad en el Perú, estimación de los años de vida saludables perdidos 2012. Lima: USAID [Internet]. 2012; Disponible en: http://www.dge.gob.pe/portal/docs/tools/Cargaen fermedad2012.pdf

4. Ministerio de Salud del Perú. Impacto socioeconómico de la Tuberculosis en el Perú 2010: documento tecnico. 2012;156.

5. Suárez Ponce CY. Ahdrencia al tratamiento y su relación con la participación de la familia en pacientes con tuberculosis en un centro de salud, 2014 [Tesis para optar el Título Profesional de licenciada en Enfermería]. [Lima - Perú]: Universidad Nacional Mayor de San Marcos; 2015.

6. Iparraguirre Usquiano MC. Factores asociados con la adherencia al tratamiento en personas afectadas con tuberculosis en los establecimientos de salud de La Microred El Porvenir Trujillo 2015 [Tesis para optar el Título de Licenciado en Enfermería]. [Trujillo - Perú]: Universidad César Vallejo; 2016.

7. González Estévez EJ. Factores asociados al abandono y adherencia al tratamiento antituberculoso en pacientes detectados en la unidades operativas del Área de Salud $\mathrm{N}^{\circ} 1$ de la Ciudad de Tulcán Ibarra - Imbabura - Ecuador; esta terapia, así como el desconocimiento de su duración.

Asimismo se encuentra relación entre factores relacionados con el paciente y adherencia terapéutica, como es la motivación del paciente con el cumplimiento del tratamiento, en el estudio de González (7), dentro de los factores encontró en los pacientes que abandonaron el tratamiento de tuberculosis, $7 \%$ por la lejanía del establecimiento de salud, $12 \%$ no le toma importancia al tratamiento, $18 \%$ de los pacientes manifestaron en su estudio, que el seguimiento no fue tan bueno, y que un $7 \%$ nunca recibió seguimiento.

Suárez y Catherine (5), en su estudio, los pacientes que tienen un buen nivel de adherencia al tratamiento, se debe tanto por las indicaciones médicas, así como el apoyo familiar, Iparraguirre (6), muestra que como factor principal a la adherencia terapéutica, son las medidas preventivas.

2012 [Tesis para optar el Título Profesional de licenciada en Enfermería]. [Ibarra, Imbabura Ecuador]: Universidad Técnica del Norte; 2017.

8. Morisky D, Green L, Levine D. Validez concurrente y predictiva autoinformada de adherencia a la medicación. Med Care. 1986; 24: 67-74.

9. Murcia Montaño MM. Evaluación de la adherencia al tratamiento para tuberculosis en el departamento del Amazonas; 2009-2012 [Tesis para optar el título de Magister en Salud Pública]. [lquitos, Perú]: Universidad Nacional de la Amazonía Peruana; 2014

10. Garnica Camacho B, Paz Zabala A, Zurita Canaviri MD, Prado Sejas G, Romero Jaldin B. Adherencia al Tratamiento de Tuberculosis en el Centro de Salud Temporal. Revista Científica Ciencia Médica. 2014;17(1):11-4.

11. Araujo Ruiz V, Arévalo Arévalo SK, Torres Ahuanari RM. Factores biosociales asociados a la adherencia al tratamiento por tuberculosis pulmonar en dos establecimientos de salud Iquitos - 2014 [Tesis para optar el Título Profesional de licenciada en Enfermería]. [lquitos, Perú]: Universidad Nacional de la Amazonía Peruana; 2014

12. Zeledón Zeledón PI, Blandón Tinoco HI, Bonilla Siu ER, Salinas AJ. Factores relacionados al cumplimiento y abandono del tratamiento antituberculoso en pacientes en león, Nicaragua / Factors related to compliance and abandonment of antituberculosis treatment in patients in León, Nicaragua / fatores associados à adesão. Journal health npeps. 29 de abril de 2017;2(1):148-60.

13. Navarro Quintero C, Rueda Rincón JL, Mendoza Ojeda JL. Factores asociados al abandono del tratamiento antituberculoso en pacientes con TB. Revista Ciencia y Cuidado. julio de 2013;10(1):19-27. 